\title{
INJECTIVE DIMENSION OF QUATERNION ORDERS
}

\author{
MARK RAMRAS
}

\begin{abstract}
Tarsy has shown that if $R$ is a discrete valuation ring with quotient field $K$, and $\Sigma$ is a quaternion $K$-algebra, then the finitistic global dimension of any $R$-order in $\Sigma$ is 1 . In this paper we allow $R$ to be any regular local ring of dimension $n$ and study the $R$-free orders $\Lambda$ in $\Sigma$. First we show that the finitistic global dimension of $\Lambda$ is $n$. Our main result concerns the injective dimension of $\Lambda$ (considered as either a left or a right $\Lambda$-module). Let $\mathfrak{M}$ denote the maximal ideal of $R$. Then the injective dimension of $\Lambda$ is $n$, unless $\Lambda / \mathfrak{M} \Lambda$ is a commutative local ring whose socle is not principal. In this case, the injective dimension of $\Lambda$ is $\infty$.
\end{abstract}

Let $(R, \mathfrak{M})$ be a regular local ring with quotient field $K$ and let $\Sigma$ be a quaternion $K$-algebra (i.e. a central simple $K$-algebra with $[\Sigma: K]=4$ ). Tarsy [5, Theorem 7] has proved that when $R$ is a discrete valuation ring (i.e. $\mathrm{gl} \operatorname{dim} R=1$ ) the finitistic global dimension ( $\mathrm{gl} \mathrm{dim}$ ) of any $R$-order in $\Sigma$ is 1 . The hypothesis that $[\Sigma: K]=4$ is crucial, since Tarsy exhibits an $R$-order in $M_{q}(K)$, the ring of $q \times q$ matrices over $K$, whose global dimension is $q-1$.

A fairly trivial generalization of Tarsy's theorem is

Proposition 3. Suppose $\mathrm{gl} \operatorname{dim} R=n$ and $\Lambda$ is an $R$-free order in $\Sigma$. Then $\mathrm{f} g \mathrm{l} \operatorname{dim} \Lambda=n$.

The main purpose of this note, however, is to extend Tarsy's proof to a determination of the self-injective dimension of an $R$-free order in $\Sigma$.

TheOREM 5. Suppose $\mathrm{gl} \operatorname{dim} R=n$ and $\Lambda$ is an $R$-free order in $\Sigma$. Then inj $\operatorname{dim}_{\Lambda} \Lambda=n$ if $\Lambda / \mathfrak{M} \Lambda$ is not a commutative local ring. If $\Lambda / \mathfrak{M} \Lambda$ is a commutative local ring, then inj $\operatorname{dim}_{\Lambda} \Lambda=n$ or $\infty$ according as the socle of $\Lambda / \mathfrak{M} \Lambda$ is, or is not, principal.

Various examples are given, including one of an order $\Lambda$ which does have infinite self-injective dimension.

Received by the editors June 19, 1972.

AMS (MOS) subject classifications (1970). Primary 16A46, 16A48, 16A90.

Key words and phrases. Order, quaternion algebra, injective dimension, Frobenius algebra, radical, socle.

(c) American Mathematical Society 1973 
LEMma 1. Let $(R, \mathfrak{M})$ be a regular local ring of dimension at least 2 . Let $\Lambda$ be an $R$-free order in $\Sigma$. Then there exists an $x$ in $\mathfrak{M}-\mathfrak{M}^{2}$ such that $\Lambda_{(x)} / x \Lambda_{(x)}$ is a quaternion algebra over $R_{(x)} / x R_{(x)}$ and $\Lambda / x \Lambda$ is an $R / x R$-free order in $\Lambda_{(x)} / x \Lambda_{(x)}$.

Proof. Let $h(\Lambda / R)$ be the homological different of $\Lambda$ over $R$ (see [1, p. 378]). Now $h(\Lambda / R) \otimes_{R} K=h(\Sigma / K)$ and the latter is nonzero because $\Sigma$ is a central separable $K$-algebra. So $h(\Lambda / R)$ is a nonzero ideal of $R$, and is thus contained in only finitely many minimal nonzero prime ideals of $R$. There are infinitely many distinct prime ideals of the form $(z), z$ in $\mathfrak{M}-\mathfrak{M}^{2}$. Hence there is one, say $(x)$, which does not contain $h(\Lambda / R)$. Therefore $\Lambda_{(x)}$ is central separable over $R_{(x)}$ and so $\Lambda_{(x)} / x \Lambda_{(x)}$ is central separable (=central simple) over $R_{(x)} / x R_{(x)}$. Since $\Lambda$ is $R$-free, $\Lambda / x \Lambda$ is $R / x R$-free and is easily seen to be an $R / x R$-order in $\Lambda_{(x)} / x \Lambda_{(x)}$. Finally, $4=[\Sigma: K]=$ $\operatorname{rank}_{R} \Lambda=\operatorname{rank}_{R / x R} \Lambda / x \Lambda=\left[\Lambda_{(x)} / x \Lambda_{(x)}: R_{(x)} / x R_{(x)}\right]$.

LEMMA 2. Let $S$ be a left noetherian ring and let $x$ be a central nonzero divisor contained in $\operatorname{Rad} S$, the Jacobson radical of $S$. Then left $\mathrm{f} g \mathrm{dim} S=$ $1+$ left $\mathrm{f} g \mathrm{dim} S / x S$.

Proof. Let $A$ be a left $S / x S$-module of finite projective dimension (p.d.) Then p.d.S $A=1+$ p.d.S/xS $A$ [3, Theorem 5.3]. Hence left f gl $\operatorname{dim} S \geqq 1+$ left $\mathrm{f} \mathrm{gl} \operatorname{dim} S / x S$.

For the reverse inequality, suppose $M$ is a left $S$-module with p.d. $S=t$. Let $0 \rightarrow L \rightarrow F \rightarrow M \rightarrow 0$ be exact with $F S$-free. Then p.d.s $L=t-1$ and $x$ is regular on $L$. Thus by [3, Theorem 5.6] we have p.d.S/xS $L / x L=t-1$, so $t-1 \leqq$ left $\mathrm{f} g l \operatorname{dim} S / x S$, i.e. $t \leqq 1+$ left $\mathrm{f} g l \operatorname{dim} S / x S$. Therefore left $\mathrm{f} g \mathrm{dim} S \leqq 1+$ left $\mathrm{f} g \mathrm{dim} S / x S$, and so the equality is established.

Proposition 3. Suppose $\mathrm{gl} \operatorname{dim} R=n$ and $\Lambda$ is an $R$-free order in $\Sigma$. Then $\mathrm{f} g \mathrm{dim} \Lambda=n$.

Proof. By induction on $n$. The case $n=1$ is Tarsy's result [5, Theorem 7]. Now assume $n>1$ and the theorem is true for $n-1$. By Lemma 1 , for some $x \in \mathfrak{M}-\mathfrak{M}^{2}, \Lambda / x \Lambda$ is an $R / x R$-free order in the quaternion algebra $\Lambda_{(x)} / x \Lambda_{(x)}$. Since gl $\operatorname{dim} R / x R=n-1$, by the induction hypothesis $\mathrm{f} g \mathrm{gl} \operatorname{dim} \Lambda / x \Lambda=n-1$. The desired result now follows from Lemma 2 .

Lemma 4. Let $F$ be a field and $S$ an F-algebra such that $[S: F]=4, S$ is quasi-local, and $S$ is noncommutative. Suppose that for every $z \in S-F$, $[F[z]: F]=2$. Then $S$ is a Frobenius algebra. In particular, $S$ is quasiFrobenius, i.e. $\operatorname{inj} \operatorname{dim}_{S} S=0$.

Proof. By hypothesis, $S / \operatorname{Rad} S$ is a simple artin ring of dimension at most 4 over $F$. If $S$ is not local, i.e. if $S / \operatorname{Rad} S$ is not a division ring, then 
$S / \operatorname{Rad} S \approx M_{2}(F)$, the $\operatorname{ring}$ of $2 \times 2$ matrices over $F$. Hence $[S / \operatorname{Rad} S: F]=$ $4=[S: F]$ and so $\operatorname{Rad} S=0$. But then $S \approx M_{2}(F)$ which is Frobenius since the trace map yields an associative, nondegenerate bilinear form $M_{2}(F) \times$ $M_{2}(F) \rightarrow F$, namely $(x, y) \backsim \rightarrow \operatorname{tr}(x y)$.

We may assume, therefore, that $\operatorname{Rad} S \neq 0$ and that $S$ is local, so that $\operatorname{Rad} S$ is the unique maximal left ideal and the unique maximal right ideal of $S$. Thus if $z$ is a nonunit, then $z \in \operatorname{Rad} S$. Since $S$ is $\operatorname{artin}, \operatorname{Rad} S$ is nilpotent. Hence $z$ is nilpotent and since $[F[z]: F]=2, z^{2}=0$.

Suppose that $\operatorname{Rad} S$, as a left ideal, is principal, say $\operatorname{Rad} S=S x$. Then $x S \subset S x$ and so $(\operatorname{Rad} S)^{2}=(S x)(S x)=S(x S) x \subset S x^{2}=0$. Therefore $\operatorname{Rad} S$ is the unique nonzero left (right) ideal of $S$. But then left $\operatorname{ann}(\operatorname{Rad} S)=$ right $\operatorname{ann}(\operatorname{Rad} S)=\operatorname{Rad} S$ and $[\operatorname{ann}(\operatorname{Rad} S): F]+[\operatorname{Rad} S: F]=2+2=$ $[S: F]$. Thus by [2, p. 414, Theorem 6.13], $S$ is Frobenius.

Let us now assume that $\operatorname{Rad} S$ is not principal. Clearly, then, $[\operatorname{Rad} S$ : $F]>1$. We claim that $[\operatorname{Rad} S: F]=3$. For suppose $[\operatorname{Rad} S: F]=2$. Let $x \in \operatorname{Rad} S, x \neq 0$. Since $S x \subsetneq \operatorname{Rad} S,[S x: F]=1$, and thus for all $s \in S$, $s x$ and $x$ are $F$-dependent. Hence $s x=a x$ for some $a \in F$. Thus $(s-a) x=0$, so $s-a$ is a nonunit and hence belongs to $\operatorname{Rad} S$. Therefore $S=F+\operatorname{Rad} S$, which implies that $[S: F]=3$, a contradiction. Thus $[\operatorname{Rad} S: F]=3$, and since $\operatorname{Rad} S \subsetneq F+\operatorname{Rad} S$, it follows that $F+\operatorname{Rad} S=S$. We claim that $\operatorname{Rad} S$ has an $F$-basis of the form $\{x, y, x y\}$. Suppose not. Then for every pair of $F$-independent elements $x$ and $y$ in $\operatorname{Rad} S, x y=a x+b y$ for some $a, b \in F$. Since $z^{2}=0$ for every $z \in \operatorname{Rad} S$, left multiplication by $x$ yields $0=b x y$, and right multiplication by $y$ yields $0=a x y$. If $x y \neq 0$, then $a=b=0$. But then $x y=0 \cdot x+0 \cdot y=0$. Thus the product of any two $F$-independent elements of $\operatorname{Rad} S$ is 0 . On the other hand, if $z, w \in \operatorname{Rad} S$ and $w=a z$ for some $a \in F$, then $z w=a z^{2}=0$. Thus $(\operatorname{Rad} S)^{2}=0$, and so $\operatorname{Rad} S$ is commutative. Hence the centralizer of $\operatorname{Rad} S$ in $S$ contains $F+\operatorname{Rad} S=S$. So $\operatorname{Rad} S \subset$ center of $S$. Thus $S=F+\operatorname{Rad} S \subset$ center of $S$, so $S$ is commutative, which is a contradiction. Therefore $\operatorname{Rad} S$ must have an $F$-basis $\{x, y, x y\}$. Hence $\{1, x, y, x y\}$ is an $F$-basis for $S$. Now $0=(x+y)^{2}=x^{2}+y^{2}+x y+$ $y x=x y+y x$. So $y x=-x y$. Thus $S=F[1, x, y, x y]$, with the defining relations $x^{2}=y^{2}=0$, and $y x=-x y$. Since $S$ is not commutative, $y x \neq x y$, and so $\operatorname{char}(F) \neq 2$.

It is easy to see that every left ideal and every right ideal of $S$ is twosided. Furthermore, the proper ideals are precisely $\operatorname{Rad} S=(x, y)$, $(\operatorname{Rad} S)^{2}=(x y)$ (the unique minimal ideal), and $(a x+b y), a, b \in F . \mathrm{A}$ simple calculation shows that each ideal $I$ is the left (and right) annihilator of some ideal $J$. Specifically, ann $(x, y)=(x y)$, ann $(x y)=(x, y)$, and for all $a, b \in F$, ann $(a x+b y)=(a x+b y)$. Furthermore, we see that for each ideal $I, \quad[\operatorname{ann}(I): F]+[I: F]=4=[S: F]$. Hence by $[2$, Theorem 6.13], $S$ is Frobenius. 
Remark. None of the four hypotheses of Lemma 4 can be dropped. We show this with four examples.

(i) $S=F[X, Y, Z] /(X, Y, Z)^{2}, \quad X, Y, Z$ independent indeterminates.

$S$ is a commutative local ring, $[S: F]=4$, and for every $w \in S-F$, $[F[w]: F]=2$. Socle $(S)=(\bar{X}, \bar{Y}, Z)$ is not principal so $\operatorname{inj} \operatorname{dim}_{S} S \neq 0$. (Since $S$ is a local artin ring, inj $\operatorname{dim}_{S} S=\infty$.)

$$
S=\left\{\left(\begin{array}{lll}
a & 0 & 0 \\
b & a & 0 \\
c & d & a
\end{array}\right) \mid a, b, c, d \in F\right\}=F\left[1, e_{21}, e_{31}, e_{32}\right] .
$$

Clearly $S$ is noncommutative and $[S: F]=4$.

$$
\operatorname{Rad} S=\left\{\left(\begin{array}{lll}
0 & 0 & 0 \\
b & 0 & 0 \\
c & d & 0
\end{array}\right) \mid b, c, d \in F\right\}
$$

and $S / \operatorname{Rad} S \approx F$, so that $S$ is local. Let

$$
w=\left(\begin{array}{lll}
0 & 0 & 0 \\
1 & 0 & 0 \\
0 & 1 & 0
\end{array}\right)=e_{21}+e_{32} .
$$

Then $w^{2}=e_{31} \neq 0$, but $w^{3}=0$. So $[F[w]: F]=3$.

If inj $\operatorname{dim}_{S} S=0$, then for all left ideals $I, 1$. ann(r. ann $\left.(I)\right)=I$. Now let $I=S e_{31}$. Then $\mathrm{r} . \operatorname{ann}(I)=\left(e_{31}, e_{32}\right) S=\operatorname{Rad} S$, and $1 . \operatorname{ann}(\operatorname{Rad} S)=S e_{21}$. Hence $I \varsubsetneqq 1$. ann (r. ann $(I))$, and so inj $\operatorname{dim}_{S} S \neq 0$.

$$
S=\left\{\left(\begin{array}{lll}
a & 0 & 0 \\
b & d & 0 \\
c & 0 & d
\end{array}\right) \mid a, b, c, d \in F\right\}=F\left[1, e_{11}, e_{21}, e_{31}\right]
$$

Again, $S$ is noncommutative and $[S: F]=4$. However $S / \operatorname{Rad} S \approx F \oplus F$, so $S$ is not quasi-local. If

$$
z=\left(\begin{array}{lll}
a & 0 & 0 \\
b & d & 0 \\
c & 0 & d
\end{array}\right)
$$

then $z^{2}-(a+d) z+a d=0$, so if $z \notin F,[F[z]: F]=2$.

Now r. ann $\left(S e_{21}\right)=S\left(e_{11}, e_{21}, e_{31}\right)=$ r. ann $\left(S e_{31}\right)$, although $S e_{21} \neq S e_{31}$. 
Hence for at least one of these ideals $I, I \neq 1$. ann(r. ann $(I))$. Thus inj $\operatorname{dim}_{S} S \neq 0$.

(iv) $S=\left\{\left(\begin{array}{llll}a & 0 & 0 & 0 \\ 0 & a & 0 & 0 \\ b & f & a & 0 \\ c & d & 0 & a\end{array}\right) \mid a, b, c, d, f \in F\right\}=F\left[1, e_{31}, e_{41}, e_{32}, e_{42}\right]$.

Note that $[S: F]=5 . S$ is noncommutative and local. $(\operatorname{Rad} S)^{2}=0$. Hence if $z \in S$ and $z$ has $a$ 's on its main diagonal, then $z-a \in \operatorname{Rad} S$, so $(z-a)^{2}=0$. Hence $[F[z]: F] \leqq 2$. Since $(\operatorname{Rad} S)^{2}=0$, if $w \in \operatorname{Rad} S$ then r. $\operatorname{ann}(S w)=\operatorname{Rad} S=$ r. $\operatorname{ann}(\operatorname{Rad} S)$. Since $S e_{31} \subsetneq \operatorname{Rad} S, \operatorname{inj} \operatorname{dim}_{S} S \neq 0$.

We come now to the main result of this paper.

THEOREM 5. Suppose $\mathrm{gl} \operatorname{dim} R=n$ and $\Lambda$ is an $R$-free order in the quaternion $K$-algebra $\Sigma$. Then inj $\operatorname{dim}_{\Lambda} \Lambda=n$ if $\Lambda / \mathfrak{M} \Lambda$ is not a commutative local ring. If it is, then inj $\operatorname{dim}_{\Lambda} \Lambda=$ either $n$ or $\infty$, according as the socle of $\Lambda / \mathfrak{M} \Lambda$ (i.e. ann $(\operatorname{Rad} \Lambda / \mathfrak{M} \Lambda)$ ) is, or is not, principal.

Proof. By induction on $n$. Let $n=1$. Passage to the completion $(\hat{R}, \hat{\mathfrak{M}})$ of the local ring $(R, \mathfrak{M})$ alters none of the hypotheses. Furthermore, if $\mathfrak{M}=(x)$, then $\operatorname{inj} \operatorname{dim}_{\Lambda} \Lambda=1+\operatorname{inj} \operatorname{dim}_{\Lambda / x \Lambda} \Lambda / x \Lambda=\operatorname{inj} \operatorname{dim}_{\hat{\Lambda}} \hat{\Lambda}$. The second equality holds because $\Lambda / x \Lambda=\Lambda / \mathfrak{M} \Lambda \approx \hat{\Lambda} / \mathfrak{M} \hat{\Lambda}=\hat{\Lambda} / x \hat{\Lambda}$. So assume now that $R$ is complete. Then if $\Lambda$ has more than one maximal two-sided ideal, by Tarsy's proof of [5, Theorem 7] $\Lambda / x \Lambda \approx \Gamma / x \Gamma$ where $\Gamma=(\stackrel{R}{R} \stackrel{(x)}{R})$. But gl $\operatorname{dim} \Gamma=1$, so inj $\operatorname{dim}_{\Gamma} \Gamma=1$. Hence inj $\operatorname{dim}_{\Lambda / x \Lambda} \Lambda / x \Lambda=$ inj $\operatorname{dim}_{\Gamma / x \Gamma} \Gamma / x \Gamma=0$, and so inj $\operatorname{dim}_{\Lambda} \Lambda=1$.

So assume that $\Lambda$ has just one maximal two-sided ideal. Thus $\Lambda / x \Lambda=$ $\Lambda / \mathfrak{M} \Lambda$ is quasi-local and $[\Lambda / \mathfrak{M} \Lambda: R / \mathfrak{M}]=\operatorname{rank}_{R} \Lambda=4$. If $z \in \Lambda-R$ then $[K[z]: K]=2$ since $\Sigma$ is a quaternion algebra. Since $R$ is a discrete valuation ring and $R[z] \subset \Lambda$, and $\Lambda$ is $R$-free, it follows that $R[z]$ is $R$-free of rank 2. Hence for any $\bar{z} \in \Lambda / \mathfrak{M} \Lambda-R / \mathfrak{M},[R / \mathfrak{M}[\bar{z}]: R / \mathfrak{M}]=2$. Thus by Lemma 4 , if $\Lambda / \mathfrak{M} \Lambda$ is not commutative, inj $\operatorname{dim}_{\Lambda / \mathfrak{M} \Lambda} \Lambda / \mathfrak{M} \Lambda=0$. Therefore inj $\operatorname{dim}_{\Lambda} \Lambda=1$.

On the other hand, if $\Gamma=\Lambda / \mathfrak{M} \Lambda$ is commutative and local, then $\Gamma$ is self-injective if and only if $\operatorname{ann}(\operatorname{Rad} \Gamma)=\operatorname{socle}(\Gamma)$ is principal. Otherwise inj $\operatorname{dim}_{\Gamma} \Gamma=\infty$. Hence inj $\operatorname{dim}_{\Lambda} \Lambda=1$ or $\infty$, according as $\operatorname{socle}(\Lambda / \mathfrak{M} \Lambda)$ is, or is not, principal.

Now assume that $n>1$ and that the theorem is true for $n-1$. By Lemma 1 , for some $x \in \mathfrak{M}-\mathfrak{M}^{2}, \Lambda / x \Lambda$ is an $R / x R$-free order in a quaternion algebra. Gl $\operatorname{dim} R / x R=n-1$ so if $\Lambda / \mathfrak{M} \Lambda$ is not commutative and local, by our induction hypothesis inj $\operatorname{dim}_{\Lambda / x \Lambda} \Lambda / x \Lambda=n-1$, and thus inj $\operatorname{dim}_{\Lambda} \Lambda=n$. If $\Lambda / \mathfrak{M} \Lambda$ is commutative and local then $\operatorname{inj} \operatorname{dim}_{\Lambda / x \Lambda} \Lambda / x \Lambda=n-1$ or $\infty$ 
according as socle $(\Lambda / \mathfrak{M} \Lambda)$ is, or is not, principal. Thus $\operatorname{inj} \operatorname{dim}_{\Lambda} \Lambda=n$ or $\infty$ according as socle $(\Lambda / \mathfrak{M} \Lambda)$ is, or is not, principal.

We conclude this paper with 3 examples which show that each of the situations described in Theorem 5 does in fact occur.

EXAMPLE A. Let $(R, \mathfrak{M})$ be a discrete valuation ring and let $\Lambda=$ $M_{2}(R)$. Then $\Lambda / \mathfrak{M} \Lambda=M_{2}(R / \mathfrak{M})$, which is noncommutative and quasilocal but not local. inj $\operatorname{dim}_{\Lambda} \Lambda=\mathrm{gl} \operatorname{dim} \Lambda=\mathrm{gl} \operatorname{dim} R=1$.

EXAmple B. Let $R=Z / 2 Z[[t]]$, the ring of formal power series in one variable over $Z / 2 Z$. Let $\Lambda=R[1, \alpha, \beta, \alpha \beta] \subset M_{2}(R)$ where

$$
\alpha=\left(\begin{array}{cc}
1+t & 1 \\
1+t+t^{2} & 1+t
\end{array}\right) \quad \text { and } \beta=\left(\begin{array}{cc}
1 & 1 \\
1+t & 1
\end{array}\right) \text {. }
$$

If we identify each $r \in R$ with $r \cdot I$, where $I$ denotes the $2 \times 2$ identity matrix, we obtain the relations $\alpha^{2}=\beta^{2}=t$, and $\alpha \beta=\beta \alpha+t^{2} .1, \alpha, \beta$, and $\alpha \beta$ are linearly independent, so $\Lambda$ is $R$-free of rank 4 and $\Lambda$ is an $R$-order in $M_{2}(K)$, where $K$ is the quotient field of $R$.

$\Lambda / t \Lambda=Z / 2 Z[1, \bar{\alpha}, \bar{\beta}, \bar{\alpha} \bar{\beta}]$ where $\bar{\alpha}^{2}=\bar{\beta}^{2}=0$ and $\bar{\alpha} \bar{\beta}=\bar{\beta} \bar{\alpha}$. Hence $\Lambda / t \Lambda \approx$ $Z / 2 Z[X, Y] /\left(X^{2}, Y^{2}\right)$, which is a commutative local ring. $\operatorname{Socle}(\Lambda / t \Lambda)=$ $\Lambda / t \Lambda(\bar{\alpha} \bar{\beta})$, so $\Lambda / t \Lambda$ is self-injective. Hence $\operatorname{inj} \operatorname{dim}_{\Lambda} \Lambda=1$.

EXAMPLE C. Let $R$ be a discrete valuation ring with maximal ideal $\mathfrak{M}=$ $(t)$. Let $\Lambda=\left\{\left(\begin{array}{cc}a & b t \\ c t & d\end{array}\right) \in M_{2}(R) \mid a \equiv d(\bmod (t))\right\}$. In other words, $\Lambda=$ $R\left[1, t e_{11}, t e_{12}, t e_{21}\right]$.

First we show that $\Lambda$ is local. Map $\Lambda$ onto $R / \mathfrak{M}$ by $\left(\begin{array}{cc}a & b t \\ c t & d\end{array}\right) \backsim \rightarrow a+\mathfrak{M}$. The kernel of this ring homomorphism is $\left\{\left(\begin{array}{cc}a & b t \\ c t & d\end{array}\right) \mid a, d \in(t)\right\}=I$ which is contained in $\operatorname{Rad} \Lambda$. Thus $\Lambda / \operatorname{Rad} \Lambda$ is a homomorphic image of $\Lambda / I \approx R / \mathfrak{M}$. Hence $I=\operatorname{Rad} \Lambda$ and $\Lambda$ is local. Next we check that $\Lambda / t \Lambda$ is commutative. Let $\alpha=t e_{11}, \beta=t e_{12}, \gamma=t e_{21}$. Then $\alpha^{2}=t \alpha, \beta^{2}=\gamma^{2}=0, \alpha \beta=t \beta, \gamma \alpha=t \gamma$, and $\alpha \gamma=\beta \alpha=0$. Also, $\beta \gamma=t \alpha$ and $\gamma \beta=t(t-\alpha) . \Lambda / t \Lambda=R /(t)[1, \bar{\alpha}, \bar{\beta}, \bar{\gamma}]$. From the above relations it follows that $\operatorname{Rad}(\Lambda / t \Lambda)=\Lambda / t \Lambda(\bar{\alpha}, \bar{\beta}, \bar{\gamma})$ and $(\operatorname{Rad} \Lambda / t \Lambda)^{2}=0$. Thus $\Lambda / t \Lambda$ is commutative and socle $(\Lambda / t \Lambda)=\operatorname{Rad}(\Lambda / t \Lambda)$, which is not principal. So by the preceding theorem, inj $\operatorname{dim}_{\Lambda} \Lambda=\infty$.

\section{REFERENCES}

1. M. Auslander and $\mathrm{O}$. Goldman, The Brauer group of a commutative ring, Trans. Amer. Math. Soc. 97 (1960), 367-409. MR 22 \#12130.

2. C. W. Curtis and I. Reiner, Representation theory of finite groups and associative algebras, Pure and Appl. Math., vol. 11, Interscience, New York, 1962, MR 26 \#2519.

3. I. Kaplansky, Commutative rings, Queen Mary College Mathematics Notes, 1966.

4. ——_ Commutative rings, Allyn and Bacon, Boston, Mass., 1970. MR 40 \#7234.

5. R. Tarsy, Global dimension of orders, Trans. Amer. Math. Soc. 151 (1970), 335-340. MR 42 \#3125.

Department of Mathematics, Boston College, Chestnut Hill, Massachusetts 02167 